\title{
Performance Evaluation of 5 Part Analyzer ADVIA 2120i at Medical College Laboratory
}

\author{
Sachin Sadanand Kale and Neha Amrut Mahajan* \\ ${ }^{1}$ Central Lab, MGM Medical College and Hospital, Auranagabad (India)
}

\begin{abstract}
Background: Central Lab, MGM Medical College is a NABL accredited medical college hospital laboratory. Lab serves more than 100 clinicians associated with hospital, hospital partners, and private consultants. We commissioned 5 part analyzer Advia 2120 i for giving better service to our clinicians and patients. Over next 2 yearsas our quality control and patient data was accumulating, we assessed the analyzer for precision, stability, differential capabilities, slide review rates.

Methods: Three hundred samples were assessed for differential and morphology flagging on each analyzer using the reference 100 cell manual differential for comparison. Precision data was collected from 3 level internal quality control samples from BioRad, and Bias was assessed from EQAS program of Randox and Biorad Laboratories

Result: Stability studies at 24 hours showed that Advia gave good reproducibility of retained patient samples, with minimal changes in the mean corpuscular volume (MCV) and hematocrit. Study showed that precision and bias of Advia 2120i never exceeded 5\% for any of CBC parameter, differential count using paired ' $t$ ' test for comparing manual countand machine generated count showed no significant difference between the two when checked for all the blood cells like neutrophils ( $\mathrm{p}: 0.3)$, lymphocytes (p:7.5), eosinophils ( $\mathrm{p}: 0.6)$, monocytes (p:0.04). Correlation for RBC flags like macrocytes, microcytes, anisocytosis and NRBC's was $100 \%$ whereas correlation for LUC was $80 \%$, blast $70 \%$,immature granulocytes $96 \%$ and atypical cells was $95 \%$.
\end{abstract}

Conclusion: In conclusion, our study reveals that performance of Advia $2120 \mathrm{i}$ is satisfactory i.e. well within the CLIA limits for CBC and the machine is fit for purpose.

Keywords: Study 5 Part Analyser, Complete Blood Count (CBC), Correlation, Slide, Flagging, Slide Review Rates.

\section{Introduction}

CBC is comparatively inexpensive but powerful diagnostic tool which provides myriads of useful information about haematological and non haematological diseases. Over the years; $\mathrm{CBC}$ analysis has evolved from manual methods to 3 part, to 5 part and lastly to 7 part Automated Counters. Like most other labs in India, we at MGM Medical College and Hospital, Aurangabad, have used 3 part cell counter, over last 10 years.

The newer 5 part analysers promise white cell differential counts, relative percent or absolute number and reticulocyte analysis, in addition to parameters given by3 partanalysers. In ADVIA 2120i whole blood is mixed with BASO reagent that contains acid and surfactant. The red cells are hemolysed and WBCs are then analysed using 2 angle scatter signals using flowcytochemistry method. The RBC's \& platelets are analyzed by the single RBC detector using the signals from a common detector with two different gain settings. Hemoglobin $(\mathrm{Hb})$ is analyzed by the modification of the manual cyanmethemoglobin method developed by ICSH. Analysis data is displayed on the Information Processing Unit (IPU). ${ }^{[1]}$
As these 5 part counters assureall these advantages with its unique flagging systems, high level of precision and accuracy, and high throughput we decided to upgrade to 5 part Analyser ADVIA 2120 for our laboratory. Objective of this study was to assess thisanalyser for precision, bias, stability, linearity, differential capabilities and accuracy of flagging ability.

Importance of this study comes from fact that laboratory should verify the claims of the manufacturer regarding all the above parameters, which is well emphasized in ISO 15189 standard $^{[2]}$ Previously many authors have done similar study, we wanted to replicate the study and check the analyser that we are using against these studies. ${ }^{[3,4]}$

\section{Materials and Methods}

As a part of performance evaluation, we assessed AD2120i by assessing its precision,bias,sample stability, differential capabilities and flagging ability as follows ${ }^{2}$

Precision: Precision is defined as closeness of agreement between results of successive measurements obtained under identical conditions. ${ }^{\mathbf{5 , 6}}$ 
Precision was monitored by daily internal quality control(IQC) procedure, Biorad controls were run thrice a day with a gap of $8 \mathrm{hrs}$. Valueswere plotted in LJ charts and monthly coefficient of variation (C.V) and standard deviation(S.D) were calculated using Unity software from Bio-rad.

Bias: Bias is used to express numerically the degree of trueness, "trueness being the closeness of agreement between the average value obtained from a large series of measurements and the true value". Bias is an average deviation from a true value.,

Bias was calculated by participating in External quality control (EQAS). EQAS for CBC was done every month by using BIORAD and $\mathrm{Z}$ score provided, was used as a measure of bias.

Sample Stability: Sample stability and the ability of the analyser to cope with these sample types is important. We assessed it by processing the same patient sample on day 1 and day 2.The sample which was processed on day1 was preserved at 2 to 8 degree $\mathrm{C}$ and rerun the next day. The values are compared and percent difference calculated. ${ }^{4}$

\section{Differential Capabilities}

Differential capabilities were analysed by using 300 consecutive patient samples on a random day. Blood films were made on each sample and a complete morphological assessment was performed.This process included 2 experienced pathologists each performing 100 cell differential.The machine results,were compared with manual differential counts, using regression analysis. We used a paired ' $t$ ' test for comparing manual and machine generated differential count by entering data in excel spreadsheet.

\section{Flagging Ability}

Flagging ability of the analyser was checked by using 300 random patient samples.

Table 1: Precision
Slides of these samples were manually reviewed and findings were compared with the flags generated by the analyser. False positive rates, False Negative rates, Positive and Negative Predictive Values were calculated as per standard methods.

\section{Result}

Precision was determined over a period of two years from June 2013 to May 2015. The mean precison is given in table 1 .As can be seen from table 1 none of the parameters have precision over $5 \%$.

Bias was also calculated over the same time period of two years (Table 2) and it was found that it never exceeded 5\% as can be seen from table 2 .

Sample stability was good and reproducibility of results was good as there was no significant difference in the readings, when compared with CLIA limits as mentioned on https://www.westgard.com/clia.htm (Retrieved on 23.12.16)(Table 3)

\section{Differential Capabilities}

After analyzing the data using a paired ' $t$ ' test for comparing manual and machine generated differential count, we found that there was no significant difference between the two when checked for all the blood cells like neutrophils (p:0.3), lymphocytes (p:7.5), eosinophils (p:0.6), monocytes (p:0.04).

\section{Flagging Ability}

RBC Flags showed $100 \%$ correlation for macrocytes,microcytes, anisocytosis, large platelets,platelet clumps,NRBC's andWBC flags like shift to Left showed $100 \%$ correlation. And correlation was $95 \%$ for atypical cells, $80 \%$ for large unstained cells,immature granulocytes and $70 \%$ for blasts.(Table 4 )

\begin{tabular}{|c|c|}
\hline Parameter & Precision \\
\hline HB & $1.9 \%$ \\
\hline RBC COUNT & $2.6 \%$ \\
\hline MCV & $1.48 \%$ \\
\hline MCH & $1.45 \%$ \\
\hline MCHC & $2.5 \%$ \\
\hline WBC COUNT & $3.49 \%$ \\
\hline PLATELET COUNT & $4.1 \%$ \\
\hline
\end{tabular}


Table 2: Bias

\begin{tabular}{|c|c|}
\hline Parameter & Bias \\
\hline HB & 0.09 \\
\hline RBC COUNT & 1.5 \\
\hline MCV & 0.2 \\
\hline MCH & 1.78 \\
\hline MCHC & 5.33 \\
\hline WBC COUNT & 1.31 \\
\hline PLATELET COUNT & 4.87 \\
\hline
\end{tabular}

Table 3: Sample stability

\begin{tabular}{|c|c|}
\hline Parameter & Aging study \% Difference at 24 hours \\
\hline HB & 1.51 \\
\hline RBC COUNT & 1.31 \\
\hline MCV & 0.5 \\
\hline MCH & 0.3 \\
\hline MCHC & 0.1 \\
\hline WBC COUNT & 0.19 \\
\hline PLATELET COUNT & 0.07 \\
\hline
\end{tabular}

Table 4:Flagging ability and percent correlation

\begin{tabular}{|c|c|}
\hline FLAGS & $\%$ CORRELATION \\
\hline MACRO & $100 \%$ \\
\hline MICRO & $100 \%$ \\
\hline ANISO & $100 \%$ \\
\hline LARGE PLATELETS & $100 \%$ \\
\hline PLATELET CLUMPS & $100 \%$ \\
\hline NRBCS & $100 \%$ \\
\hline LUC & $80 \%$ \\
\hline BLASTS & $70 \%$ \\
\hline SHIFT TO LEFT & $100 \%$ \\
\hline IG & $96 \%$ \\
\hline ATYPICAL CELLS & $95 \%$ \\
\hline
\end{tabular}

\section{Discussion}

ADVIA 2120i haematology analyser is claimed to be designed to improve workflow efficiency for high volume laboratories through enhanced flagging and elimination of preanalytical sample preparation and sorting. The results of this evaluation demonstrate the analyser's performance for its precision, bias, sample stability, differential capabilities and flagging ability.

None of the CBC parameters exceeded $5 \%$ for precision, and were well within CLIA limits..Our study showed this shows machine's precision is very good. Similar findings were reported by $\mathrm{G}$ Bourner et al. ${ }^{4} \mathrm{Bias}$ for the parameters was also less than 5\%, thereby assuring its accuracy. As bias and precision is good the reliability of results generated by the machine is very good.

Sample stabilityand reproducibility of results was good, as there was no significant difference in the readings, when compared with CLIA limits. Only proviso was that samples are received in standard transport and storage conditions. This assures us that we can have fairly good and accurate results even when samples are received from distant places.

Our analysis shows that differential capabilitiesof machine were fairly accurate when compared to manual methods and can be acceptedwithout any doubt. So the slide review 
and time spent by the pathologist in this activity was significantly reduced.

RBC flags like microcytes, macrocytes and anisocytosis correlated $100 \%$ with manual review and were accurate, hence they can be accepted without hesitation. Flags for NRBC's were correlated well on manual review, most of these cases were from newborn samples and haemolytic anemias. False positive flags were noted for LUC (20\%),atypical lymphocytes (5\%) and blasts $(30 \%)$. These flags lead to slide review; for all flagged cases, manual verification made sure that doubtful cases were scrutinised carefully and not missed. False positive flags are better than false negative because risk of missing a significant abnormality is less that way. And no false negative flags were noted in our study.

In a study from South Korea by Sue Jun Kim et al false negative rate, false positive rate, sensitivity and specificity were $14.3 \%, 11.3 \%, 41 \%$ and $85 \%$ respectively for ADVIA 2120i.Giacomini et al in their study showed imprecision was low in all the parameters evaluated,stability study showed no significant change and carry over was negligiblie. ${ }^{10}$ This is comparable to our study. Velizarova. $\mathrm{M}$ et al in their study showed that accuracy of ADVIA 2120i was excellent for all CBC and white cell differential parameters. ${ }^{11}$ Our study also came to same conclusion

\section{Conclusion}

Reproducibility and accuracy for ADVIA 2120i is within acceptable limits. Differential capabilities are very reliable and one can depend on machine generated data. Ageing studies for samples gave accurate results provided samples were accepted in proper storage and transport conditions. We conclude that the ADVIA2120i gives accurate and precise $\mathrm{CBC}$ and 5 part differential results and is an excellent upgrade over 3 part cell counters, and it is well suited for medium to large sized hospital laboratories.

\section{Reference}

1. ADVIAi2120Haematologyanalyser Operating manual.

2. International Standard.ISO 15189:2012.Medical Laboratories-requirements for quality and competence. $3^{\text {rd }}$ ed,2012.

3. Tracey Fernandez, Lynn BessertDomack, Dinah Montes, Richard Piniero, Eileen Landrum, Esther Vital.Performance Evaluation of the Coulter LH 750 Hematology Analyser. Laboratory Hematology 7.2001.p217-228.

4. GBourner, J Dhaliwal, J Sumner. Performance Evaluation of the Largest Fully Automated Hematology Analysers in a Large, Commercial Laboratory Setting: a 4 Way, Side by Side Study. Laboratory Hematology II:I-xx;p1-16.

5. DouglasChesher. Evaluating assay precision.ClinBiochem Rev Vol 29 Suppl (i)August 2008;p 23-26.

6. Marry A Williamson, L Michael Snyder.Wallach's Interpretation of Diagnostic Tests. $9^{\text {th }}$ ed. Philadelphia, USA. Lippincott Wiliamsand Wilkins; Wolter Kluwer Health.2011

7. RogerJohnson.Assessment of Bias with emphasis on Method Comparison. ClinBiochem Rev Vol 29Suppl(i) August 2008;p37-42.

8. Richard A, McPherson, Mathew R. Pincus.Henry's Clinical diagnosis and Management by Laboratory //methods,21/e. Virginia,USA.Saunders;Elsevier:2008.

9. Sue Jung Kim, Yoonjung Kim, Saeam Shin, Jaewoo Song, Jong Rakchoi. Comparison study of the rates of Manual peripheral blood smear review from 3 automated hematology analysers, UnicelDxH 800,ADVIA 2120i; and XE 2100,Using international Consensus group Guidelines. ArchPathol Lab Med Vol 136,November 2012;p1408-1413.

10. A.Giacomini,PLegovini,F.Antico,G.Gessoni,S.Valdverde. Evaluation of Platelet Analysis on ADVIA120 Hematology System. Laboratory Haematology 7;2001;p180-185.

11. Velizarova $M$, Yacheva $T$, Tzatchev $K$. Evaluation of analytical reliability of hematology analyser ADVIA2120i; verification and comparison with COULTER LH 750.Meditsinski Pregled/Medical Review 2012 Vol.48 no 2;p36-43.

*Corresponding author:

Dr Neha Amrut Mahajan, 41, Atharva, besides water tank, Vedant Nagar, Railway station road, Aurangabad, Maharshtra, India-431005.

Phone: +910240 6601100

Email: nehaamahajan@yahoo.com

Date of Submission : 29.12.2017

Date of Acceptance : 10.01.2017

Financial or other Competing Interests: None.

Date of Publication : 25.01.2018 